\title{
VIRTÙ E VIZI DELLE BANCHE E DEI BANCHIERI. UNA NOTA INTRODUTTIVA
}

\author{
SILVIO BERETTA (*)
}

SunTO. - Banche e banchieri sono spesso stati oggetto di apprezzamenti polemici: uomini politici, economisti, biografi e opinionisti hanno messo infatti in evidenza le conseguenze potenzialmente negative dei loro comportamenti sul benessere delle persone e sulla prosperità delle economie. Le conseguenze dei fallimenti a catena dei banchieri fiorentini nel quattordicesimo secolo sull'economia reale sono un esempio significativo di tale negativo collegamento. Vengono successivamente prese in esame le idee di Marx, di Schumpeter e di Keynes sulla funzione delle banche e dei banchieri. Le argomentazioni svolte da Keynes nel "Treatise on Money" appaiono, a questo riguardo, di particolare significato e attualità. Ai nostri giorni infine, e con riferimento all'economia italiana, gli assetti regolatori definiti dall'Unione Europea danno origine a ulteriori problemi, date le differenze tuttora esistenti fra i sistemi finanziari dei paesi dell'Unione e fra i rispettivi assetti istituzionali.

$$
* * *
$$

ABSTRACT. - Banks and bankers are often the subject of controversial appreciations: politicians, economists, biographers and commentators have put it out the potentially negative consequences of their behavior on the welfare of the people and the prosperity of the economies. The consequences of the failures of Florentine bankers in the fourteenth century on the real economy are a significant example of such a negative connection. The ideas of Marx, Schumpeter and Keynes on the function of banks and bankers are then examined. The arguments developed by Keynes in its "Treatise on Money" appear, in this regard, of particular significance. At the present time, finally, and with reference to the Italian economy, the European Union's regulatory arrangements give rise to further problems, owing to the differences that still exist among the financial systems of the EU countries and their institutional settings.

(*) Presidente dell'Istituto Lombardo Accademia di Scienze e Lettere, Milano; Università degli Studi di Pavia, Italia. E-mail: info@istitutolombardo.it 
Nell'aprire questo incontro di studio, per la partecipazione al quale sono grato ai colleghi che hanno accettato di prendervi parte e al Direttore della Banca d'Italia di Milano che si è reso disponibile a portare in questa sede gli orientamenti dell' "Ente regolatore" del mercato, non posso non sottolineare preliminarmente quanto agevole sia rinvenire negli scritti di personalità di spicco, e non necessariamente economisti, opinioni assai radicali che avvalorano il ruolo di "croce" sistematicamente esercitato, nella loro opinione, dall'attore "banche", e dai banchieri in particolare, nei confronti di una collettività. Già Thomas Jefferson, uno dei padri fondatori degli Stati Uniti d'America di cui fu Segretario di Stato e due volte Presidente, affermava: "Io credo che le istituzioni bancarie siano più pericolose per le nostre libertà di quanto non lo siano gli eserciti permanenti. Se il popolo americano permetterà mai alle banche private di controllare l'emissione del denaro, dapprima attraverso l'inflazione e poi con la deflazione, le banche e le compagnie che nasceranno intorno alle banche priveranno il popolo dei suoi beni finché i loro figli si ritroveranno senza neanche una casa sul continente che i loro padri hanno conquistato"'. Ancora negli anni '70 Federico Caffè poteva pervenire a una conclusione analoga argomentando nei seguenti termini: "Da tempo sono convinto che la sovrastruttura finanziario-borsistica, con le caratteristiche che presenta nei paesi capitalisticamente avanzati, favorisca non già il vigore competitivo, ma un gioco spregiudicato di tipo predatorio che opera sistematicamente a danno di categorie innumerevoli e sprovvedute di risparmiatori, in un quadro istituzionale che, di fatto, consente e legittima la ricorrente decurtazione o il pratico spossessamento dei loro peculi" ${ }^{2}$. Anche Ron Chernow, biografo di grandi banchieri (categoria della quale per altro preconizzava la scomparsa), si domandava, in apertura di un saggio del 1997, "...is there any creature on God's green earth so unloved as a banker?" per aggiun-

1 Jefferson faceva riferimento al progetto - poi realizzato - di attribuire a un soggetto di diritto privato il potere di emissione. Per una recente disamina della dura contrapposizione che vide schierati, da una parte, Thomas Jefferson (Segretario di Stato) e dall'altra Alexander Hamilton (Segretario al Tesoro) e che vide la vittoria del secondo dopo un' aspra battaglia parlamentare che si concluse - nel 1791 - con la nascita della First Bank of the United States con sede a Filadelfia, cfr. G. BOTTARO, Alexander Hamilton: potere politico e potere economico in America in "Il Politico", maggio-agosto 2012, pp. 15-32.

2 F. CAFFE, Un'economia in ritardo. Contributi alla critica della recente politica economica italiana, Torino, Boringhieri, 1976, p. 18. 
gere: "We envision bim in stereotypical form as a grim, bumorless man in late middle age with iron-gray hair, wirerimmed spectacles, and a costive disposition. Of somber mien, with a permanent scowl on his face, he wears the dark, monochromatic suits of a small-town funeral director"' ${ }^{\text {. Avvici- }}$ nandoci ulteriormente ai giorni nostri, vale la pena di segnalare la domanda che si (e ci) rivolgeva Massimo Mucchetti, opinionista e parlamentare, quando si chiedeva: "Ma che mondo è questo nostro nel quale la concentrazione della ricchezza è tale per cui i bonus della Goldman Sachs, anno domini 2009, sono pari al reddito di 224 milioni delle persone più povere del pianeta?"4.

Della cattiva fama delle banche e dei banchieri, tuttavia, non si sono occupati soltanto uomini di Stato, economisti ed esperti ma anche, fra gli altri, filosofi e anarchici. Due riferimenti fra i tanti. Si racconta ad esempio (è Guido Carli a ricordarcelo ${ }^{5}$ ) che Schopenauer, visitando una galleria nella quale erano esposti i ritratti dei Fugger - banchieri tedeschi fra il $14^{\circ}$ e il $17^{\circ}$ secolo e finanziatori degli Asburgo - avrebbe esclamato: "Quando guardo i vostri volti, mi accorgo che Dio non è con voi". Seppure in tutt'altro contesto, analoghi dovevano essere i sentimenti che animavano l'anarchico italiano Mario Buda - amico di Sacco e Vanzetti - quando, il 16 settembre 1920, fece esplodere un carro trainato da un cavallo e imbottito di pezzi di ferro e di esplosivo nelle

3 R. CHERnOw, The Death of the Banker. The Decline and Fall of the Great Financial Dynasties and the Triumph of the Small Investor, New York, Vintage Books, 1997, p. 3. Di Chernow sono noti, in particolare, i saggi The House of Morgan: An American Banking Dynasty and the Rise of Modern Finance (Atlantic Monthly Press, 1990) e The Warburgs: The Twentieth-century Odyssey of a Remarkable Jewish Family (New York, Random House, 1993).

4 M. MucchetTi, "Corriere della Sera", 29 ottobre 2011. Dalle ricerche di Michael Kumhof e Romain Rancière del Fondo Monetario Internazionale, di Robert Frank della Cornell University e di Lars Osberg della Dalhouise University di Halifax Mucchetti ricava argomenti per istituire una relazione fra aumento della diseguaglianza di redditi e ricchezza, aumento dell'indebitamento dei ceti medio-bassi della popolazione favorito da un sistema bancario che dall'aumentata diseguaglianza trae risorse aggiuntive da impiegare, aumento delle sofferenze bancarie, interventi pubblici di "salvataggio" e, conseguenza ultima, aumento del debito pubblico.

5 G. CARLI, Perchéle banche sono impopolari in "Bancaria", 1976 n.11, ristampato nel volume collettaneo Economia E Banca tra cronaca e storia, Roma, Bancaria Editrice, 2008, pp. 395-408. Sulla vita di Jacob Fugger, fondatore dell'omonima dinastia, cfr. G. SteInMETZ, The Richest Man Who Ever Lived, 2015 by Greg Steinmetz (trad. it. Il creatore di re, Milano, Baldini\&Castoldi, 2016). 
immediate vicinanze della sede della J.P. Morgan \& Company a Wall Street mentre vi si stava tenendo una importante riunione, provocando decine di morti e centinaia di feriti ${ }^{6}$. Bersaglio principale dell'attentato era lo stesso banchiere Morgan, per altro assente dalla riunione: era stato comunque a quest'ultimo che si era fatto ricorso perché intervenissse a salvare i mercati finanziari in occasione della crisi del $1907^{7}$. Nemmeno i fatti della storia, tuttavia, favoriscono i banchieri e la loro fama. Una sequenza assai significativa di eventi negativi è, ad esempio, quella rievocata da Carlo M. Cipolla nella monografia dedicata alle vicende dell'economia fiorentina nella prima metà del Trecento, saggio che a sua volta trae molte informazioni dalla Cronica di Giovanni Villani ${ }^{8}$. Vale la pena di dedicare qualche parola ai fatti di quel tempo. Dopo un prolungato periodo di prosperità, il verificarsi pressoché simultaneo 1) della bancarotta del Comune di Firenze, che nel febbraio 1345 dichiarò di non poter fare fronte alle proprie obbligazioni, con conseguente crollo del corso dei titoli, 2) dello stato di insolvenza di re Edoardo III d'Inghilterra, il quale non fu più quindi in grado di onorare i propri debiti nei confronti dei banchieri fiorentini e 3) della corsa al prelievo dei fondi detenuti presso le banche fiorentine dai grandi depositanti napoletani, dovuto al timore di un cambio di campo da parte di Firenze, dallo schieramento guelfo a quello ghibellino, fece sì che "[...] si arrivò fatalmente alla rovina. Dopo di aver stentatamente cercato per qualche anno di far fronte a una situazione sempre più difficile, nel 1343 fallivano i Peruzzi e tre anni più tardi, nel 1346, fallivano i Bardi. E fu la catastrofe [...] In questi casi una bancarotta tira l'altra per cui dopo i Peruzzi ed i Bardi saltarono gli Acciaiuoli, i Bonaccorsi, i Cocchi, gli Antellesi, i Corsini, i da Uzzano, i Perendoli. Era tutto il

6 L'episodio, il primo nella storia degli attentati realizzati con la tecnica dell'autobomba, è rievocato in M. DAVIS, Buda's Wagon. A Brief History of the Car Bomb, London-New York, Verso, 2007 (trad.it. Breve storia dell'autobomba. Dal 1920 all'Iraq di oggi. Un secolo di esplosioni, Torino, Einaudi, 2007, pp. 3-6).

7 Sul ruolo di John Pierpont Morgan in quella crisi cfr. R.F. BRUNER, S.D. CARR, The Panic of 1907. Lessons Learned from the Market's Perfect Storm, Hoboken, New Jersey, John Wiley \& Sons, 2007, passim. Per un affascinante profilo della personalità di Morgan banchiere e collezionista cfr., oltre alla biografia di Chernow citata nella nota 3, il saggio di H. TuZZI, Morte di un magnate americano, Skira editore, 2013.

8 C.M. CiPOLla, Il fiorino e il quattrino. La politica monetaria a Firenze nel 1300, Bologna, il Mulino, 1982, passim e in particolare pp. 9-28. 
Gotha della finanza e dell'economia fiorentine che finiva davanti ai giudici fallimentari. Il crollo delle banche travolse tutti coloro che vi tenevano depositi e che, nei migliori dei casi, riebbero soltanto la metà o il terzo o addirittura il quinto dei loro depositi [...] Una massa notevole di ricchezza andò così distrutta...Né questo fu tutto. La bancarotta delle compagnie provocò sconquasso anche nei settori secondario e terziario, direttamente e indirettamente: direttamente perché le compagnie, oltre all'attività bancaria, esercitavano anche attività mercantile e manifatturiera; indirettamente perché i loro fallimenti provocarono sul mercato una drastica contrazione del credito. Una volta scoppiata la crisi, si mise in moto un perverso meccanismo di moltiplicatore alla rovescia per cui la crisi alimentò se stessa e si estese a macchia d'olio"'.

Prescindendo poi sia dalle espressioni a effetto sia da casi clamorosi del passato, anche l' "alta teoria" conosce la centralità del ruolo della banca e del banchiere, con tutti i problemi che da tale ruolo conseguono. La conosce ad esempio Marx, per il quale l'attività del banchiere "...consiste nel concentrare nelle sue mani e in grandi masse il capitale monetario disponibile per il prestito, così che di fronte ai capitalisti industriali e commerciali, in luogo del singolo individuo che dà denaro a prestito, si trovano i banchieri, come rappresentanti di tutti coloro che danno denaro a prestito. Essi diventano gli amministratori generali del capitale monetario. D'altro lato essi rappresentano, di fronte a tutti coloro che danno a prestito, la figura di chi prende a prestito, poiché essi prendono a prestito per tutto quanto il mondo commerciale" ${ }^{10}$. E la conosce anche Schumpeter il quale, nel delineare la propria teoria dello sviluppo economico imperniata sulla figura dell' "impren-

9 C.M. CIPOLla, ivi, passim e in particolare pp. 9-28. Il tema della crisi fiorentina verrà in seguito ripreso dallo stesso Cipolla, precisamente nel capitolo dal titolo "Uomini duri" del saggio Tre storie extra vaganti (Bologna, il Mulino, 1994, pp. 9-47), con particolare riguardo alle vicende del Banco de' Bardi e dei suoi esponenti fino dalla creazione, nel 1310, della "Compagnia di Lapo e Doffo de'Bardi": ancora negli anni '20 del Trecento il Banco poteva contare su circa venticinque filiali con agenti, uffici e magazzini dislocati in tutta Europa e oltre, da Avignone a Bruges, da Costantinopoli a Gerusalemme, da Marsiglia a Parigi a Siviglia a Tunisi a Venezia.

10 K. MARX, La moneta e il credito. Raccolta di scritti (a cura di Suzanne de Brunhoff e Pierre Ewenczyk), Milano, Feltrinelli, 1981, p. 279. Il volume raccoglie e ordina scritti marxiani su denaro, moneta e credito redatti in tempi diversi, secondo criteri esposti dai curatori nelle pp. 9-59. Il passo citato è tratto dal Libro terzo de $I l$ Capitale: per i criteri di scelta seguiti cfr. in particolare pp. 243-4. 
ditore innovatore" 11 , così descrive l'attività del banchiere: "Il banchiere...non è tanto un intermediario della merce 'potere d'acquisto', ma un 'produttore' di questa merce. Dal momento però che oggi, normalmente, anche tutti i fondi di riserva e i risparmi affluiscono a lui e che su di lui si concentra la domanda totale di potere d'acquisto disponibile, sia esistente sia da creare, egli ha per così dire sostituito o messo sotto la sua tutela i capitalisti privati, è diventato egli stesso il capitalista. Egli sta fra coloro che vogliono introdurre nuove combinazioni e i possessori dei mezzi di produzione. Egli costituisce in sostanza un fenomeno dello sviluppo, ma solo laddove non esista una autorità centrale che dirige il processo economico della società. Egli rende possibile l'introduzione di nuove combinazioni, in certo qual modo emette a nome della società il mandato necessario per introdurle. È l'eforo dell'economia di scambio"12. Ma la conosceva già nel 1930 anche il Keynes del Treatise on Money ${ }^{13}$, indulgendo per altro anch'egli, e senza risparmio, nel gusto dell'espressione colorita e della metafora sorprendente. Vale la pena di soffermarsi un po' a lungo sui passi del Treatise che riguardano il banchiere. Innanzitutto, per Keynes "Il banchiere moderno compie...due distinti tipi di servizi. Egli offre un sostituto alla moneta di stato operando da Stanza di compensazione e trasferendo pagamenti correnti nell'ambito della sua clientela a mezzo di registrazioni a debito

11 J.A. SCHumPeTER, The Theory of Economic Development, Cambridge, Mass. Harvard University Press, 1934 (trad. it. Teoria dello sviluppo economico, Firenze, Sansoni, 1977). Nel ricollocare lo sviluppo al centro della propria analisi, in questo recuperando l'approccio classico e marxiano in contrapposizione a quello marginalista e neoclassico, Schumpeter ne fornisce tuttavia una definizione originale. Secondo Schumpeter, infatti, "Per 'sviluppo' si devono...intendere solo quei mutamenti della vita economica che non sono ad essa imposti dall'esterno, ma scaturiscono dall'interno, dalla sua propria iniziativa. Se risultasse che tali cause del mutamento, derivanti dalla sfera economica stessa, non esistono e che il fenomeno che in pratica chiamiamo sviluppo economico si fonda esclusivamente sul fatto che i dati cambiano e l'economia vi si adatta continuamente, diremmo che non esiste alcun sviluppo economico. Intendendo con ciò che lo sviluppo dell'economia non è un fenomeno che vada spiegato economicamente, ma che l'economia, di per sé senza sviluppo, viene per così dire trascinata dai mutamenti del suo ambiente, e che le ragioni e quindi la spiegazione dello sviluppo devono essere cercati al di fuori del gruppo di fatti contemplati dalla teoria economica" (p. 73 della trad. it.).

12 J.A. SCHUMPETER, op. cit., p. 83 della trad. it.

13 J.M. Keynes, A Treatise on Money, Londra, Macmillan, 1930, 2 voll. (trad, it. Trattato della moneta, Milano, Feltrinelli, 1979, 2 voll.). I passi citati di seguito sono tratti dalle pp. 416-23 della trad. it. 
e a credito. Ma agisce pure come mediatore nei confronti di un particolare tipo di mutui, in quanto riceve dal pubblico dei depositi che impiega nell'acquisto di titoli o prestando all'industria e al commercio soprattutto per sopperire ai loro fabbisogni di capitale d'esercizio. Questa dualità di funzioni costituisce la chiave di vari difficili problemi della moderna teoria della moneta e del credito...". Tali problemi vengono accentuati dalla circostanza per la quale "...in tempi moderni, le banche sono giunte a controllare risorse tanto importanti ed hanno costituito una organizzazione di così vasta portata da rendersi di gran lunga i maggiori mutuanti a breve termine. Naturalmente, perciò, esse sono giunte a sentire come un dovere, non meno importante di quello di fornire moneta, il far fronte alle fluttuanti richieste della collettività per questo genere particolare di mutui. Il dilemma della banca moderna è quello di combinare soddisfacentemente queste due funzioni. Come fornitore di moneta fiduciaria, il sistema bancario ha il dovere di preservare la prescritta base oggettiva di tale moneta. Come fornitore di prestiti a termini e condizioni particolari, il sistema ha il dovere di conciliare, nel miglior modo possibile, la sua offerta di questo tipo di prestiti alla domanda che vien fatta al saggio d'interesse d'equilibrio...La preservazione della base oggettiva è inoltre legata...in un modo peculiare... al saggio al quale il sistema bancario, nella sua qualità di mutuante, facilita gli investimenti in nuovo capitale. Giacché, per questa ragione, il completo adempimento di uno dei suoi doveri è a volte incompatibile con il completo adempimento dell'altro, sta alle persone che controllano il sistema bancario decidere quale sia il fine che è giusto prevalga o, se nessuno dei due deve prevalere in modo assoluto, realizzare un giusto compromesso".

$\grave{E}$ a questo punto che Keynes introduce la categoria degli "eretici monetari", le cui opinioni sarebbero accomunate dal convincimento "...che in qualche modo le banche possano fornire tutte le risorse reali di cui l'industria ed il commercio possono ragionevolmente avere bisogno, senza un costo reale per nessuno e che, se esse tengono a differenziare i loro crediti, lo facciano unicamente in conformità a qualche criterio relativo all'utilizzo che i mutuatari intendono fare delle risorse prese a prestito". Ne consegue che "Se la moneta...è disponibile in quantità sufficiente ed a buone condizioni non potrà sorgere difficoltà alla piena occupazione di tutta l'offerta disponibile di fattori della produzione" dal momento che "Per il singolo commerciante od industriale il 'credito bancario' significa 'capitale d'esercizio'; un prestito da parte 
della sua banca lo fornisce dei mezzi per pagare salari, per acquistare materiali e per mantenere degli stocks...Perché allora... [si chiede l'eretico, n.d.r.]...se le banche possono creare il credito, dovrebbero rifiutare una qualsiasi ragionevole richiesta di esso? E perché dovrebbero esigere un compenso per ciò che costa loro poco o nulla? I nostri disagi gli sembrano derivare dal fatto che le banche hanno monopolizzato questo potere di creare il credito così da mettersi in grado, restringendo artificialmente la sua offerta, di assegnargli un prezzo e realizzare in tal modo un profitto. Perché, se esse posseggono questo magico potere, sono così taccagne? Perché gli industriali dovrebbero avere meno capitale d'esercizio di quello che desiderano o essere costretti a pagarlo il $5 \%$ ? Non può esservi che una risposta. I banchieri, avendo il monopolio della magia, esercitano il loro potere parsimoniosamente allo scopo di elevare il prezzo. Se i fornai costituissero una corporazione chiusa che potesse fabbricare il pane con le pietre non ne seguirebbe che essi ridurrebbero il prezzo della pagnotta da 4 libbre al costo di una pietra dello stesso peso. Dove lavora la magia il pubblico non ne gode a pieno i benefici a meno che non venga nazionalizzata. Il nostro eretico ammette invero che dobbiamo aver cura di evitare 'l'inflazione', ma sostiene che questa si verifica soltanto quando viene creato del credito che non corrisponde ad alcun processo produttivo... La riforma monetaria...consiste nell'adattare la creazione del credito in modo da soddisfare tutte le richieste genuine di capitale d'esercizio...Inoltre non vi è ragione di gravare su tale credito oltre a ciò che sia richiesto per fare fronte alle sofferenze e alle spese di amministrazione".

Naturalmente Keynes è consapevole del fatto che i banchieri hanno una risposta a questo riguardo, ma ritiene che questa sia "...ben poco convincente. Il banchiere non nega infatti che, in un senso o nell'altro, egli possa creare del credito. L'unica argilla che egli chiede per questo atto di creazione è una idonea proporzione d'oro (od altra forma di riserve)...Se dobbiamo credere ai banchieri, l'importo del capitale d'esercizio disponibile per l'industria sembra così dipendere in qualche modo dall'importo dell'oro presso la Banca d'Inghilterra.... Naturalmente gli eretici replicano che questo modo di parlare dell'oro ha molto del bluff. E' ovvio che non può essere l'oro presso la Banca d'Inghilterra che mette in grado l'industria di marciare. Infatti la maggior parte di esso non viene toccata per anni e anni, e se anche dovesse svanire per l'aria, tutto potrebbe continuare come prima, sempreché non lo si sapesse...i banchieri si sforzano tuttavia di riportarsi al fatto, 
così vitale per essi, delle loro riserve, come a un sintomo se non come una causa. Per quanto siano essi che creano il credito, gli importi di credito da essi creati non sono né arbitrari né illimitati. Questi importi sono regolati dalle necessità degli affari da una parte, ma anche dallo stato delle loro riserve dall'altra. Se essi dovessero creare del credito senza riguardo alle loro riserve, l'oro fuggirebbe dal paese e metterebbe in pericolo la convertibilità della moneta; oppure, se il gold standard non è in vigore, $i$ cambi esteri salirebbero, aumentando così il costo di tutte le importazioni. Questo discorso sembra alla luce del semplice buon senso giusto e convincente ed anche l'eretico comincia a sentirsi a disagio. Ma non è convinto del tutto... Infatti come è possibile che il credito richiesto dall'industriale possa dipendere dalla quantità del metallo che sta sotto chiave nei sotterranei di Threadneedle Street?...Il principale fine di questo trattato...[il Treatise on Money, n.d.r.]...è quello di rispondere chiaramente a questi dubbi. Qual è il giusto criterio per la creazione di un credito che sia non-inflazionistico...?...Abbiamo trovato che la risposta sta nel mantenimento di un equilibrio fra il saggio del risparmio ed il valore dei nuovi investimenti. Vale a dire che $\mathrm{i}$ banchieri possono creare del credito senza prestare il fianco al sospetto di tendenze inflazionistiche soltanto se l'effetto netto di tale creazione sul valore dei nuovi investimenti non è quello di aumentare il valore di tali investimenti sopra l'importo del risparmio corrente del pubblico; e, analogamente, presteranno il fianco al sospetto di azione deflazionistica se non creano credito a sufficienza per impedire che il valore dei nuovi investimenti scenda al disotto dell'importo del risparmio corrente...la prova che tale equilibrio sia o non sia effettivamente mantenuto può sempre essere rintracciata nella stabilità o meno del livello dei prezzi della produzione complessiva. L'errore che gli eretici hanno compiuto va ricercato...nel fatto che essi non tengono conto della possibilità dell'inflazione dei profitti...non percepiscono che i prezzi possono salire anche se il saggio di remunerazione dei fattori della produzione per unità di prodotto sia invariato...Cosa dire però del criterio dei banchieri circa le loro riserve? Possiamo avere dato agli eretici una risposta convincente; essa non sembra però la stessa risposta che hanno dato i loro nemici banchieri. Infatti non è la stessa. Lo stato delle riserve dei banchieri è, effettivamente, un sintomo...che l'offerta di potere d'acquisto supera i mezzi materiali di soddisfarlo entro il paese...La caratteristica di questo criterio... sta nel fatto che in esso non si ha un indicatore della presenza o meno dell'inflazione dei profitti, ma soltanto della relazione 
più o meno appropriata in cui il grado di inflazione o di deflazione sta ai fenomeni del mondo circostante...Pertanto l'ideale della stabilità non è conseguito né con i princìpi degli eretici, né con i princìpi dei banchieri. Le pratiche dei primi possono produrre l'inflazione dei profitti, ma lo stesso può dirsi per le pratiche dei secondi con in più lo svantaggio che, in altre occasioni, esse possono anche produrre la deflazione dei profitti. Nessuno di essi afferra il giusto criterio di stabilità, cioè l'equilibrio fra il risparmio e gli investimenti...I banchieri, così stando le cose, non tentano neppure di conservare la stabilità dei prezzi e dell'occupazione...il loro obiettivo in regime di gold standard è quello di tenere il passo con il comportamento medio dei sistemi bancari del mondo nel suo complesso. La loro idea non è quella di mantenersi sobri, ma, in conformità ad un perfetto savoir vivre, di essere in quello stesso stato di ebbrezza (o magari di mal di capo) che caratterizza tutta la compagnia. Gli eretici, d'altro lato, sono tutti per una stretta sobrietà e per la temperatura normale, nell'interesse della massima attività ed efficienza dell'organismo economico. Ma le loro nozioni di fisiologia sono insufficienti ed essi non si rendono conto che per un uomo l'unico modo di mantenersi sano è quello di dosarsi con un saggio di interesse esattamente armonizzato alla temperatura, alla pressione sanguigna e alle altre circostanze di fatto. Un ulteriore malinteso, se posso continuare nelle mie metafore mediche, è suscettibile di sorgere, dopo che l'instabilità si sia approfondita e siano state iniziate le cure, per la ragione che non si tiene presente il fatto che i rimedi, la cui efficacia in via definitiva non è dubbia, non possono produrre la reazione richiesta se non dopo un certo intervallo di tempo... Spesso il risultato è che il rimedio è esagerato".

$\grave{E}$ a questo punto, per finire, che Keynes si produce in una metafora del tutto inconsueta anche per il meno convenzionale dei contesti: egli sottolinea infatti che: " $E$ ' come se la famiglia desse ad un ragazzo dosi successive d'olio di ricino di dieci in dieci minuti finché la prima dose non avesse effetto o, per prendere un migliore parallelo, è come se i diversi membri della famiglia dessero al ragazzo successive dosi ignorando ciascuno le dosi date dagli altri. Il ragazzo si ammalerà gravemente: allora sarà somministrato del bismuto con lo stesso sistema. Gli scienziati annunceranno che i ragazzi sono soggetti ad un ciclo di diarrea-stitichezza dovuto, essi aggiungeranno, al tempo oppure ad alternative di ottimismo e di pessimismo fra i membri della famiglia. Se il ciclo richiesto dalla prima dose per agire è costante, scopriranno che il ciclo 
è un ciclo vero e proprio a periodo costante. Forse suggeriranno che il rimedio è da trovarsi nella somministrazione di bismuto quando il ragazzo è stitico e di olio di ricino quando è nelle condizioni opposte. Ma più probabilmente i parenti si divideranno nel partito del bismuto e nel partito dell'olio di ricino, uno dei quali, impressionato dagli orrori della diarrea, rinuncerà all'olio di ricino e l'altro, commosso dalla depressione della stitichezza, abiurerà il bismuto. Non è cosa facile mantenersi sulla giusta via della salute continua".

Dai brani riportati, e particolarmente da quelli del Treatise, la figura del banchiere come intermediario/produttore di potere d'acquisto e di fondi per il prestito, unitamente alla centralità della sua collocazione nel sistema economico in funzione dello sviluppo di questo, emergono quindi con tutta rilevanza: questa, a sua volta, è tanto più significativa e gravida di conseguenze quanto più si rivelano strette le interconnessioni/interferenze fra le plurime funzioni che il banchiere svolge e quanto più si fa pressante, nell'esercizio di tali funzioni, la propensione dei banchieri ad agire in regime di monopolio. Tale propensione, congiuntamente ai vincoli istituzionali che disciplinano l'attività di tali soggetti, può di conseguenza rendere i banchieri stessi (in quanto aggregato di persone e di imprese) "disfunzionali" agli obiettivi di pubblico interesse rappresentati dalla piena occupazione delle risorse produttive in regime di stabilità dei prezzi: gli oneri che il mutuatario deve accollarsi in sede di acquisizione di fondi per il prestito, funzione anche del grado di monopolio che caratterizza il sistema bancario, incidono infatti in misura significativa sulla sua propensione all'investimento e quindi sulla sua disponibilità a farsi promotore di sviluppo, specialmente quando si tratti dell' "imprenditore innovatore" schumpeteriano. Il grado di percezione (o di mancata percezione) delle condizioni di equilibrio con piena occupazione, la rilevanza dei gap temporali, i vincoli formali posti dai regolatori all'esercizio della funzione bancaria, gli eventuali giochi di strategia posti in essere, nonché gli "umori" prevalenti nella comunità internazionale dei banchieri costituiscono, come si comprende dai passi citati e specialmente dagli ultimi, altrettante determinanti aggiuntive del comportamento del banchiere, quindi del suo "peso" e della natura "problematica" del suo ruolo: di conseguenza, per tornare agli inizi di questa nota, anche - per dirla con Jefferson - della sua "pericolosità".

A ricondurre tali problemi, e altri ancora, alla situazione italiana nelle circostanze attuali, e ai suoi propri problemi, ci aiuteranno i relatori che prenderanno la parola nel corso di questo incontro. Mi limito, 
per chiudere, a richiamare gli scenari problematici ulteriori aperti dai nuovi assetti regolatorio-istituzionali definiti a livello di Unione Europea. Li chiarisce assai bene Marco Lossani quando, in un recente "Quaderno" e con riferimento al Green Paper pubblicato dalla Commissione Europea il 18 febbraio 2015, sottolinea come “...l'attuale 'presunto mercato europeo dei capitali' sia in realtà estremamente frammentato", dal momento che "I diversi mercati finanziari esistenti nei diversi paesi UE sono in realtà assai differenti tra loro non solo per dimensione e grado di sviluppo, ma anche per la diversa fiscalità, per il differente diritto societario nonché per le difformi procedure fallimentari" ${ }^{14}$. Ne consegue, e qui il riferimento è a Basilea 3 e alle previste modalità di realizzazione dell'Unione Bancaria, che l'obiettivo di assicurare stabilità finanziaria a un sistema, quello europeo, finanziariamente integrato ma nel quale le politiche di regolazione e di supervisione sono stabilite ancora a livello nazionale, comporta rischi, fra i quali “...il rischio - molto concreto secondo alcuni - che il combinato disposto di queste nuove disposizioni/provvedimenti favorisca anche nella regione dell'Eurozona una crescente disintermediazione dell'industria bancaria a favore dello sviluppo dello shadow banking system" ${ }^{15}$.

14 M. Lossani, Mutamenti strutturali e il futuro delle banche italiane in M. Lossani, P. Manasse, C.A. Carnevale MafFé, Mutamenti strutturali e il futuro delle banche italiane, Associazione per lo Sviluppo degli Studi di Banca e Borsa Università Cattolica del Sacro Cuore, Quaderno n. 284, Milano, 2015, p. 10.

15 M. LossANI, ibidem, p. 9. 